\title{
Convergências das redes: nova fronteira para compreender a cibercidadania e o jornalismo on-line
}

Ricardo Nicola

\section{Resumo:}

Ainda que a constituição da internet possa parecer um tema já bastante explorado pelas pesquisas cibersociais, o assunto, contudo, emerge e se manifesta como um terreno vasto para gerar investigações das ciências sociais aplicadas, e, em especial, da comunicação - tendo como papel de destaque, o jornalismo digital. Pretende-se apontar as principais variáveis que auxiliam na compreensão dos fenômenos hipermidiáticos presentes na web. Como outrora apresentadas em outros artigos de minha autoria, tais variáveis estão categorizadas como redes em convergência que, na atualidade, totalizam-se em seis, sendo elas: redes de símbolos (signos), línguas, nações, comunidades, mercados e máquinas; e, que neste artigo, se mostram importante para compreender a cidadania online, agora percebida não só como fruto de um sistema fragmentário, mas o próprio.

\section{Palavras Chave:}

internet, cibercidadania, jornalismo digital, mídias digitais, comportamento cibersocial

\begin{abstract}
:
Although the constitution of the internet may seem a subject already well exploited by cyber social researches, the matter, however, emerges and manifests itself as a vast territory to generate applied research on social sciences and, in particular, communication - which main role is the digital journalism. It is intended to sharpen the key variables that help in understanding the hypermedia phenomena presents on the web. As a previously presented in other articles of my own authorship, such variables are categorized as convergence networks in which, in actuality, totaling up to six, which are: networks of symbols (signs), languages, nations, communities, markets and machines, and in this article, have shown important to understand the on-line citizenship, now perceived not only as a result of a fragmented system, but itself.
\end{abstract}

\section{Keywords:}

internet, cyber citizen, digital journalism, digital media, behavior cyber social, virtual community

"A arquitetura da inteligência é a arquitetura da conectividade. É a arquitetura que traz consigo os três principais ambientes espaciais em que vivemos hoje: a mente, o mundo e as redes" (1) (Derrick de Kerckhove).

A representação da cibercidadania está cada vez mais intrínseca aos movimentos sociais na rede. E esta representatividade tem sido ora manifesta através de vários sites (figura 1) - que disponibiliza serviços de auxílio a vítimas como crianças, adolescentes etc. -, ora exposta por meio de blogs ou comunidades virtuais (jornalismo digital comunitário) (figuras 2 e 3), que procuram discutir as netiquetas entre outros assuntos corelacionados à convivência no ciberespaço. 
Este artigo - como parte dos aportes teóricos da pesquisa pós-doutoral "Cibercidadania e seus discursos transculturais", realizado no McLuhan Program in Culture and Technology da Universidade de Toronto -, portanto, pretende esboçar como alguns dos principais caminhos - dentre os quais, a identificação das redes vêm auxiliando na compreensão do mundo cibercultural e, na esteira dele, a cibercidadania.

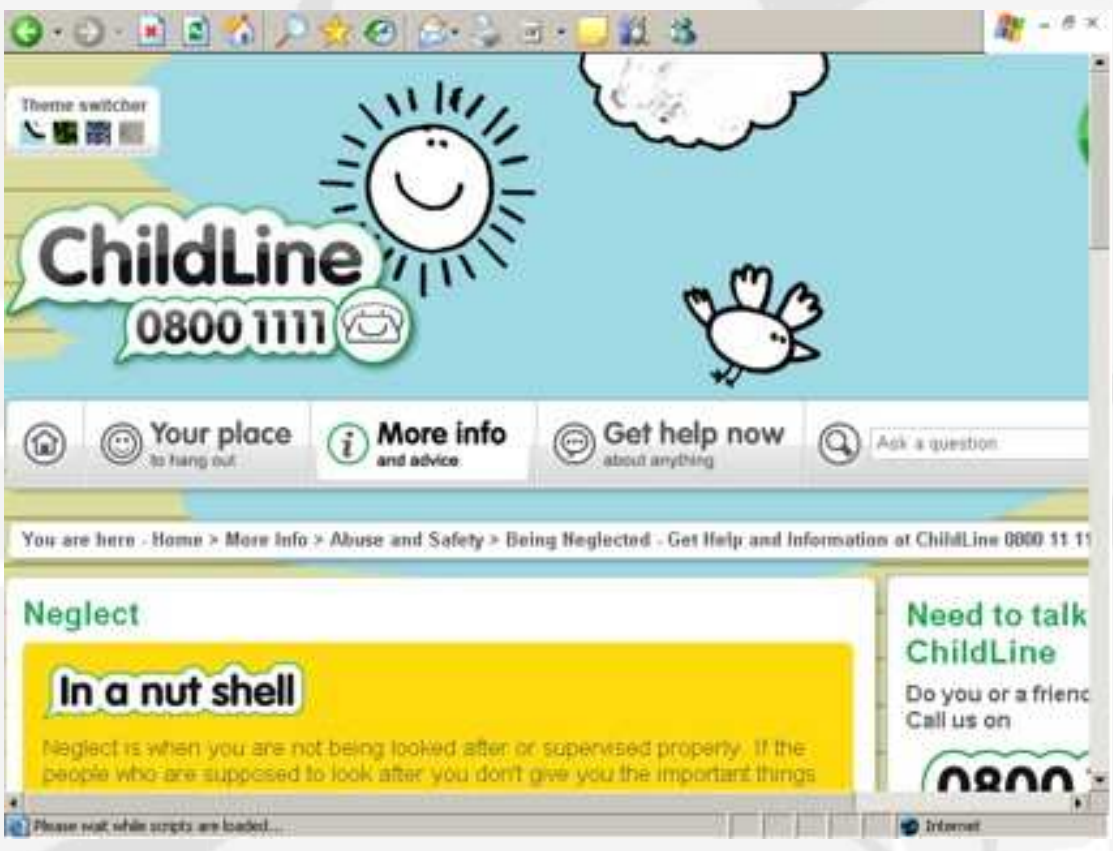

Figura 1 - Site americano responsável por auxílio on-line para crianças e adolescentes http://www.donthideit.com/profiles/darryl.html

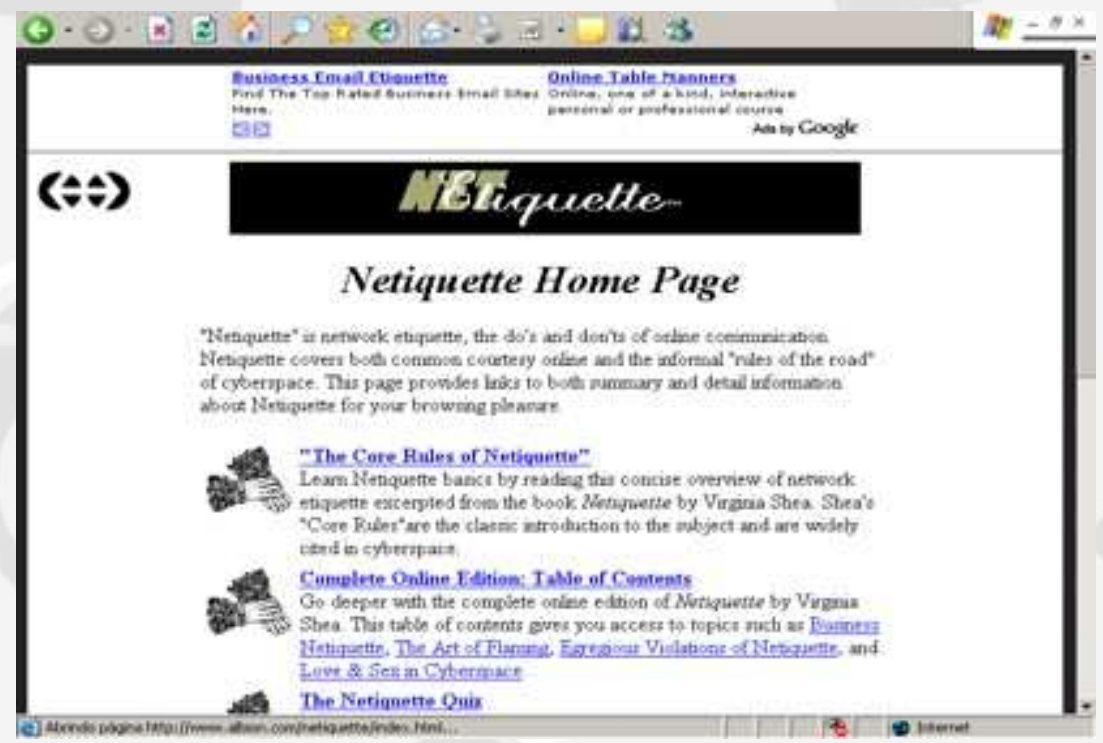

Figura 2 - Site que disponibiliza uma série de netiquetas http://www.albion.com/netiquette/index.html 


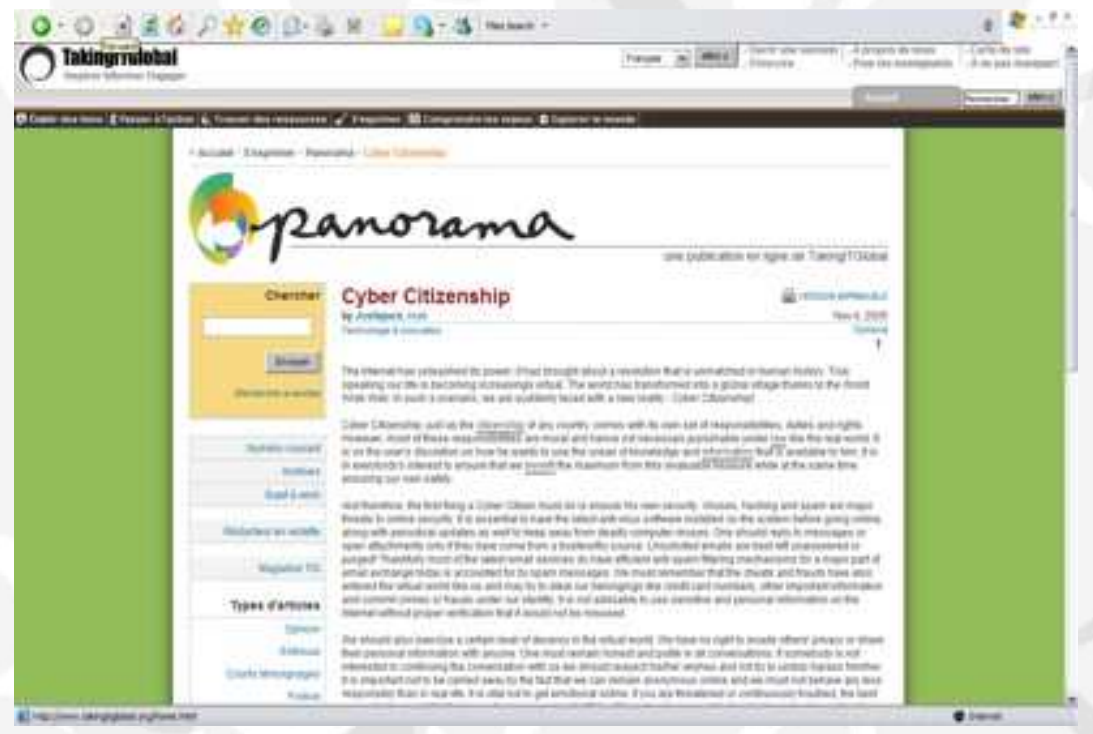

Figura 3 - Revista eletrônica Panorama

http://fr.takingitglobal.org/express/panorama/article.html?ContentID=6533\&start=0

\section{Redes de redes}

Diante do exposto, não tem sido fácil compreender como o impacto das redes à internet tem se manifestado. Muitas inferências existem em torno da questão (Lévy, 1999; Kerckhove, 2001), mas em geral, o tema diluise num discurso por vezes fragmentado, ou ainda, construído numa base em que o fenômeno das redes em convergência vem sendo desconsiderado na totalidade. Para compreender como o impacto das redes representa para a Web, é mister esboçar as mais importantes características de cada uma - identificadas até agora - e envolvidas direta e indiretamente na constituição do sistema internetworking (Rheingold, 2007) e do próprio jornalismo digital.

Pierre Lévy aproveita para pontuar seis diferentes redes na constituição do ciberespaço. Derrick de Kerckhove, no entanto, acrescenta ainda a importância da mente e do mundo. Ante a extensão do tema, cujo papel de um simples artigo não possibilitaria esgotá-lo, optou-se num primeiro momento pelo desdobramento das redes.

Conforme identificadas anteriormente, são elas, redes de símbolos (sinais), línguas, nações, comunidades, mercados, e máquinas e suas constantes interações entre si, que se convencionou por convergências. Então, em outras palavras, qual seria a importância delas e o que é exatamente novo nestas distinções para este estudo?

A princípio, é necessário definir que tipo de característica tem cada rede e o que está afetando (ou contribuindo) na construção da internet e na concepção do direito e do dever no mundo tecnológico, razão primeira dessa pesquisa pós-doutoral. Sabe-se que são vastos os atributos concedidos a cada uma delas, contudo, chamarei a atenção exclusivamente às qualidades significativas e, destarte, mais aplicadas à cibercidadania e ao jornalismo on-line.

\section{Símbolos (signos)}

A definição do que possa ser entendido por símbolos é complexa (Morin, 1999) e sua concepção primária determina um vasto campo conceitual. Logo, o símbolo pode ser objeto, personagens, figuras, sons ou cores e 
seus usos para representar idéias ou ainda conceitos.

Considerando os dados como informação, símbolos representam principalmente a menor parte, ou elemento da comunicação. Quando se estuda o impacto dos símbolos no jornalismo digital, vê-se como sua representação tem construído um grande mosaico de interação e, cuja relação, vem permitindo a existência do próprio sistema de ciberleitura. Muitos pesquisadores têm dedicado ao seu estudo, exemplos como o de Charles Sanders Peirce (1839-1914), Ferdinand de Saussure (1857-1913), Jacques Derrida, Roland Barthes, Jean Baudrillard, Algirdas Julius Greimas, e muitos outros.

Como teóricos da vida pós-moderna, eles recriaram um novo espectro dos modos de ver a internet através da teoria dos signos, ou ainda, a chamada Semiótica. Turkle chamou-os de os filósofos da Cultura de Simulação.

Neste ponto e, de forma geral, Saussure concebeu a noção do ciberespaço como não havendo uma conexão obrigatória entre o signo e o seu significado. Por conseguinte, constantemente clicamos em um número ilimitado de ícones no mundo cibernético, cuja convergência desconstrói a necessidade da existência dessa dependência para a construção e descontrução do significado. E, ainda, Saussure insiste quanto à arbitrariedade do signo, o que influenciou grandemente filósofos mais tarde, em especial os teóricos pósmodernos anteriormente citados.

Como uma rede de símbolos, o reino dos pós-modernistas se coloca e se assume como o representante maior da noção desta relação signficante-significado. Nós não podemos mais separar a idéia de ícone e da rede. É a base do seu espaço... No entanto, a fim de compreender como o significado pode ter sido edificado, a teoria de Algirdas Julius Greimas é bem-vinda, que compreende o desenvolvimento de uma versão da semiótica estrutural, conhecida como semiótica gerativa, ou seja, trata-se de uma tentativa de mudar o foco da disciplina da sinalização para a de sistemas de significação. Suas teorias contribuíram para desenvolver as concepções de Saussure, Hjelmslev, Claude Lévi-Strauss e Maurice Merleau-Ponty.

Através destas teorias sobre signos, podemos compreender quando os mesmos dominaram as formas como nos comunicamos no mundo virtual. Há muitos estudos em relação ao impacto destas teorias neste fenômeno comunicacional atual, e que se aplicam também na cibercidadania. Em outras palavras, para dar manutenção à cidadania tecnológica, é mister compreender este sistema de símbolos, como funciona, como se organiza. Em linha geral, precisamos saber que as nossas ferramentas para tentar (re)definir a cibercidadania, inicia-se com a introdução a este sistema da modernidade líquida (Baumman, 2005), caso contrário, o mundo virtual passará a ser um perfeito estranho, e as suas relações com o significado será julgado pelos pesquisadores, governos, etc, um enigma.

O mundo virtual demanda ferramentas pós-modernas e sua base tem se centralizado na filosofia da linguagem e que, também, deve ser inspirado na ecologia do signos. Mas, como já havia sido relatado anteriormente, a internet é uma complexa rede de outras redes e apreender a sua concepção completa, interliga-se à estrutura das línguas envolvidas nelas e, como tal, este será o próximo passo para a caracterização de cibercidadania e do jornalismo on-line.

\section{Línguas}

As preocupações com o uso da língua na internet não é novidade e sabemos que o inglês é o dominante entre os outros idiomas no ecossistema digital. Mesmo que o índice não represente uma queda expressiva, "no prazo de três anos deste estudo [abaixo], o percentual de web sites no respectivo idioma tenha caído para 68\%" (Pastore, 2000).

Existe, contudo, uma lista de falantes e suas línguas respectivas para as páginas da web, e sua percentagem 
tem mudado gradativamente, e, é óbvio, que o impacto econômico se faz presente. E, sem dúvida alguma, que isto tem influenciado o conceito de cibercidadania e seu processo de (re) construção, e a produção do jornalismo on-line. Portanto, pelo idioma da nação também se cria a idéia da nacionalidade; seria possível o inglês a língua dos cibercidadãos?

No seu discurso, Darin Barney (2007) chamou a atenção para o que ele intitulou de "Uma Nação Sob o Domínio do Google - Cidadania na República Tecnológico [One Nation Under Google - Citinzenship in the Technological Republic (sem tradução para o Português)]" quando o mesmo explorou o crescimento do inglês e seus usos pelo mercado midiático. Ao que Carvim (2001), corrobora sua tese com um quadro quanto à presença das línguas na internet e de seus desdobramentos:

\begin{tabular}{|c|c|c|c|c|}
\hline $\mathrm{N}$. & Língua & N. de web pages & N. de falantes (em milhares) & $\begin{array}{l}\text { Rel. falantes/ } \\
\text { web pages }\end{array}$ \\
\hline 1 & Inglês & $214,250,996$ & 322,000 & 1.5 \\
\hline 2 & Islandês & 136,788 & 250 & 1.8 \\
\hline 3 & Sueco & $2,929,241$ & 9,000 & 3.1 \\
\hline 4 & Dinamarquês & $1,374,886$ & 5,292 & 3.9 \\
\hline 5 & Norueguês & $1,259,189$ & 5,000 & 3.9 \\
\hline 6 & Finlandês & $1,198,956$ & 6,000 & 5.0 \\
\hline 7 & Alemão & $18,069,744$ & 98,000 & 5.4 \\
\hline 8 & Holandês & $3,161,844$ & 20,000 & 6.3 \\
\hline 9 & Estoniano & 173,265 & 1,100 & 6.4 \\
\hline 10 & Japonês & $18,335,739$ & 125,000 & 6.8 \\
\hline 11 & Italiano & $4,883,497$ & 37,000 & 7.6 \\
\hline 12 & Francês & $9,262,663$ & 72,000 & 7.8 \\
\hline 13 & Catalão & 443,301 & 4,353 & 9.8 \\
\hline 14 & Tcheco & 991,075 & 12,000 & 12.1 \\
\hline 15 & Basco & 36,321 & 588 & 16.2 \\
\hline 16 & Eslovênico & 134,454 & 2,218 & 16.5 \\
\hline 17 & Coreano & $4,046,530$ & 75,000 & 18.5 \\
\hline 18 & Letão & 60,959 & 1,550 & 25.4 \\
\hline 19 & Russo & $5,900,956$ & 170,000 & 28.8 \\
\hline 20 & Húngaro & 498,625 & 14,500 & 29.1 \\
\hline 21 & Português & $4,291,237$ & 170,000 & 39.6 \\
\hline 22 & Grego & 287,980 & 12,000 & 41.7 \\
\hline 23 & Espanhol & $7,573,064$ & 332,000 & 43.8 \\
\hline 24 & Lituano & 82,829 & 4,000 & 48.3 \\
\hline 25 & Polonês & 848,672 & 44,000 & 51.8 \\
\hline 26 & Hebreu & 198,030 & 12,000 & 60.6 \\
\hline 27 & Chinês & $12,113,803$ & 885,000 & 73.1 \\
\hline 28 & Turco & 430,996 & 59,000 & 136.9 \\
\hline 29 & Búlgaro & 51,336 & 9,000 & 175.3 \\
\hline 30 & Romeno & 141,587 & 26,000 & 183.6 \\
\hline 31 & Árabe & $127,565,000$ & 202,000 & $1,583.5$ \\
\hline
\end{tabular}

Fonte: Adaptado de Carvim (2001) (2).

Quadro1 - Falantes por língua (Language to web pages in that language, 2001) 
Se estudarmos as línguas assinaladas na tabela como inglês (1), russo (19), português (21), espanhol (23), chinês (27), e árabe (31), muitas razões mercadológicas explicam tais posições. Neste momento, o inglês está na primeira posição por razões que nós já detectamos, mas Warschauer demonstra a sua diminuição na próxima década como especificado abaixo:

(...) $\mathrm{O}$ acesso à internet está chegando ao ponto da saturação nos Estados Unidos, mas já está decolando em muitos outros países ao redor do mundo. Com uma massa crítica de usuários on-line em suas línguas específicas, mais e mais pessoas e empresas estão criando sites em que a língua e seus falantes é contemplada também com um número maior de parceiros em potencial para a comunicação mediada por computador. Esta tendência também se acelera pela expansão dos sistemas operacionais e aplicativos de páginas da web autorizados em scripts não-românicos, que permitem às pessoas se comunicarem em línguas não-alfabéticos com mais facilidade, tais como japonês, chinês e hebraico. Devido a estas tendências, espera-se que a proporção de sites da web em inglês venha a declinar para 40\% na próxima década (Graddol, 1997) (WARSCHAUER, 2004: 12).(3)

Entre estas 31 línguas escolhidas por este estudo, além do inglês, também o Russo, o Português, o Chinês se destacam e algumas delas apontam para as características emergentes de seus países, tais como Índia, Rússia (ex-URSS), Brasil e China, respectivamente.

Afinal de contas, o inglês - como ressaltado - é a lingua da cibercidania e deve ser otimizada nos conteúdos dos jornais on-line; no entanto, numa rápida visão, no mundo cibernético sua caracterização se alterou... passou por um processo de hibridação. Os usuários vêm criando uma nova faceta do inglês, o Globish: misto de reduções no inglês e código da linguagem global.

Assim, talvez o Globish têm sido uma resultante das formas de se expressar do não-falante inglês e os códigos de expressão internacional, interpostos pela linguagem da hibridação dos mercados dos meios de comunicação como os emoticons, os avatares, a gíria informática, etc; além disso, está em construção as particularidades da língua do cibercidadão, e acredita-se que os relacionamentos dos mais diversificados, em rede, estarão para confirmá-la, tal qual a produção de notícias on-line.

\section{Nações \& comunidades}

Através do impacto da tecnologia na sociedade, vê-se dia a dia as nações confrontarem uma nova configuração do espaço, o que representa uma reorganização das esferas sociais e políticas. Desde os fins dos anos 1980, vivemos a aldeia global prevista por McLuhan (1962), como fruto da globalização dos mercados, dos meios de comunicação social, e de telecomunicações.

Como sabemos, após este período, as sociedades têm experienciado o território não-espacial e este fato representa a base dos seus desafios; inquestionável, a tecnologia é um "produto da sociedade e a grande responsável por esta transformação, e junto com ela, nações estão adaptando a esta era da informação, e outras não". Entretanto, o ecossistema digital tem criado o conceito de nação digital como Anthony G. Wilhelm (2004) descreveu com entusiasmo:

(...) O objetivo de uma nação digital não é apenas o de fazer as coisas mais depressa, é fazê-las de maneira diferente, revolucionando a forma de como se educam pessoas, prestam-se cuidados de saúde (...) Implementar uma visão de nação digital irá resguardar do país milhares de milhões de dólares ao longo dos próximos anos e irá aumentar grandemente a qualidade de vida, pois a tecnologia amplia as possibilidades de escolha e oportunidades. Nas arenas de saúde e oportunidade do e-trabalho, por si 
só, as tecnologias de informação e de comunicação nos convidam para maximizar o alcance de todos ao nosso atendimento médico no ambiente rural, e ao alívio do tráfego (...) (WILHELM, 2004: xii). (4)

Por certo, o autor amplia a vantagem da tecnologia na sociedade. No entanto, outros países estão excluídos deste contexto e os mesmos são nações que fazem parte do fosso digital. Por outro lado, muitos governos migraram para a web; há uma lista de países e muitos deles, sem dúvida, estão no primeiro mundo.

Em 2008, um mapa de nações e seus acessos à internet foram atualizados por Miniwatts Marketing Group e está disponível em http://www.internetworldstats.com/ stats.htm e apresento, a seguir, alguns dos principais deles:

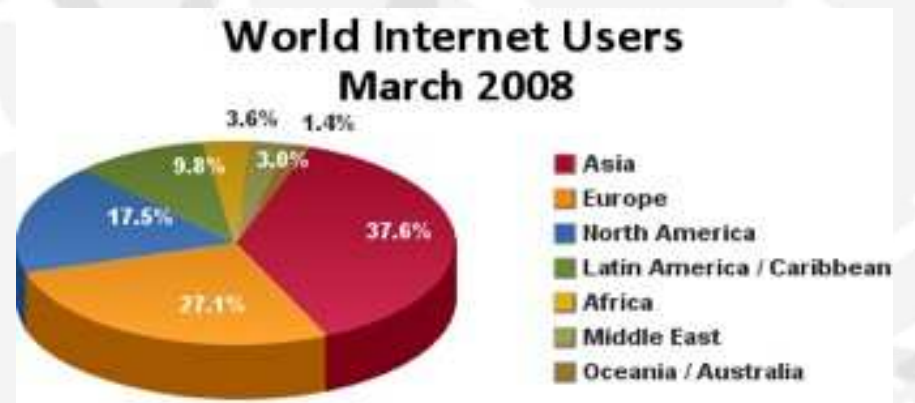

Source: mow internetworldstats com/stats htm

Copyright@ 2008, Miniwatts Marketing Group

Figura 4

\section{World Internet Penetration Rates} March 2008

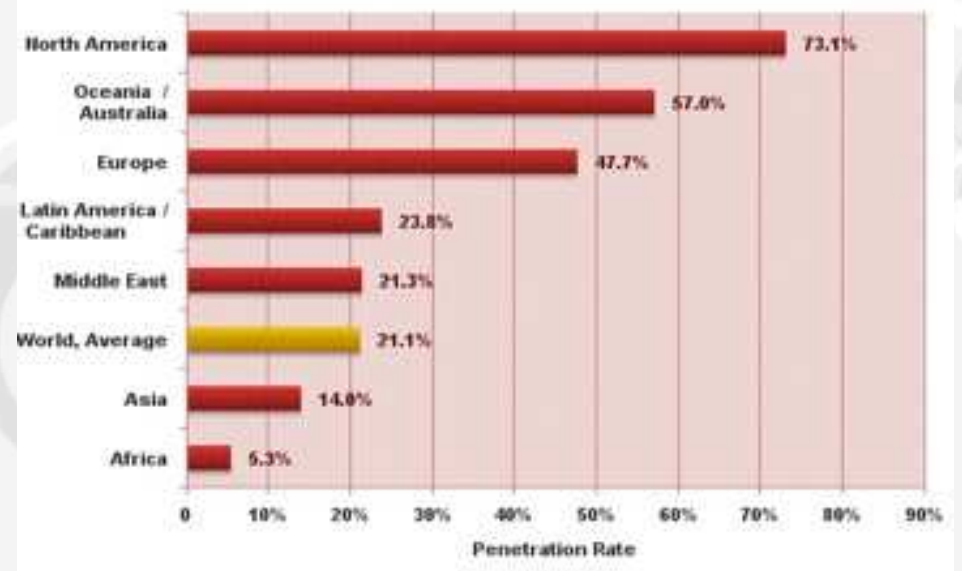

Note Penetration Rates are Dased on a world population of $6.676,120.288$ for mid-year 2008 Copvrighte 2006, Miniwatts Marketing Orovp - www internetworlostats.com

Figura 5 


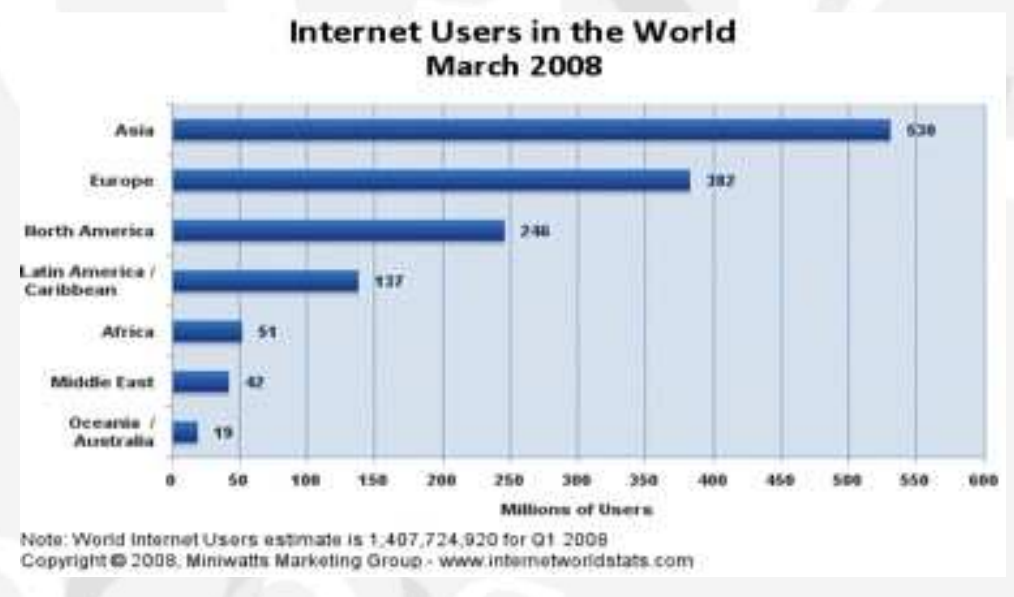

Figura 6

Estas estatísticas revelam-nos um panorama sobre a realidade on-line e apontam para um perfil do usuário atual que esboça uma verdadeira Apartheid Digital (Nicola, 2007).

Numa rápida seqüência, a conexão mente-ciberespaço ocorreu de forma automática e tem justificado o sucesso da internet como um espaço em que as manifestações transnacionalistas (Nicola, 2007) tem sido a grande bandeira dos usuários. Por conseguinte, o seu representante mais importante tem sido a comunidade, em outras palavras, a comunidade virtual. Howard Rheingold é um dos mais entusiastas dos pesquisadores envolvidos diretamente com o conceito de Comunidade Virtual (CV). Suas obras exploram não só a definiç̧ão de $\mathrm{CV}$, bem como suas raízes em gerar o desejo de construção da nacionalidade na web.

Povos e mais povos estão penetrando na ágora eletrônica com a sua cultura, seus discursos, a fim de disponibilizar, por assim dizer, suas experiências ciberculturais. E nós sabemos que este pode ser o grande desafio dos nossos tempos, dissolver o Digital Divide. Como perguntaria Benedict Anderson (1983), que tipo de nacionalidade o ciberespaço estará criando em nossas mentes, ou melhor, na mente dos usuários?

Fazer parte do ciberespaço não é se justifica necessariamente pela transferência das populações para a web... Será que ao se efetivar tal transferência, junto dela as diferenças econômicas iriam também, ou não? Será que o mundo on-line é um verdadeiro espelho para as nações? Por outro lado, é a internet espelhos, ou realmente janelas? Serve-se para tão-somente mostrar os poderes político-ideológicos de algumas nações primeiromundistas em seu aparato comunicacional para legitimar seus domínios nesta aldeia glocal (Canclini, 1999)?

Devemos nos centrar nestas questões durante a (re)construção e/ou (re)definição do ecossistema digital, em que se estabelece como um território para as nações; caso contrário, estaríamos criando uma idéia de nãoparticipação de outros povos, tornado-a apenas figurativa dentro dos desdobramentos da vida social on-line.

De forma consciente, devemos dar tempo ao tempo e pensar sobre o futuro da nação digital. É necessário ter em mente que este é um trabalho que precisamos realizar agora, ou a política on-line pode engessar as ações cibersociais das nações menos favorecidas; trata-se de objetivos claros a ser alcançados a longo prazo.

Imagine onde gostaríamos de estar em dez ou vinte anos em termos de ciberespaço, o que pode ser considerado um exercício segundo Crapton, mas assim ele o define:

Ciberespaço para nós, agora, é uma espécie de mapeamento que temos em nosso projeto, dos medos e das esperanças: é o nosso 'projeto'. Como tal, uma projeção, o ciberespaço apresenta uma série de representações (e, portanto, cartográficas) destas questões. Não é, por acaso, que todos os mapas são 
projeções. As questões não são, em sua abstração, mapeamento (a cartografia as tem mapeado há séculos), mas com a sua política espacial, o ciberespaço é um caso clássico de um espaço que é construído, e que, por sua vez produz (de forma espacializada) a subjetividade. Assim, não só se está mapeando o ciberespaço, mas, no traçado dos seus contornos estão sendo delineadas suas linhas com os nossos próprios rostos. O ciberespaço é um espaço de conhecimento geográfico, que se situa igualmente entre sociedade e tecnologia. Convida-nos o "campo" para uma crítica da política de representação espacial e que tem, como premissa, a crítica da política da geografia e do espaço (CRAPTON, 2003: 6). (5)

Podemos inferir que muitos aspectos territoriais se justificam para compreender o mundo virtual. Um deles pode redimensionar a origem dos crimes que ocorrem na mídia on-line, cuja dimensão geográfica é a sua principal característica, como aponta o Citizen Lab (Laboratório da Cidadania), na Universidade de Toronto (UofT). Mas, à medida que os cientistas políticos procuram uma razão para compreender a distribuição desequilibrada do acesso à internet dentro do sistema, suas respostas, além das consideradas óbvias, não esgotam as dúvidas apresentadas, e muitas perguntas, sem respostas, desafiam, inclusive, a idéia de geografica na web, e mais ainda, elas redirecionam, e frisam, ao sentido desterritorial do espaço.

O mundo digital é uma nação de cidadãos num território do não-lugar (Marc Augé, 1983) em que os encontros acontecem num ambiente fragmentado. Será este, portanto, um novo desafio que confunde a nossa visão das crises no ciberespaço?

Enquanto isso, em maior escala, um número significativo de CVs tem recriado a divisão entre espaços individuais e coletivos na rede. Howard Rheingold, Manuel Castells, Píerre Lévy, Marshall McLuhan entre outros ciberfilósofos e cientistas sociais debatem a importância dessa realidade comunitária. Considerado o criador da Well - uma das mais conhecidas comunidades virtuais da rede - Rheingold, que escreveu o livro A comunidade virtual e, mais tarde, Smart Mobs, destacou que as CVs tornaram-se importantes nos relacionamento em redes, e têm representado a solidificação, considerada ideal, quanto ao compartilhamento de dados.

Já Tim Jordan explica a definição de comunidades cibernéticas através da criação de avatares:

Comunidades emergem no ciberespaço, momento em que usuários criam avatares ao retornarem para o mesmo espaço informativo. As pessoas podem até achar que não estão sozinhos no ciberespaço, mas têm desenvolvido relações com uma série de outros avatares estável e tornaram-se parte de uma comunidade virtual. Comunidades virtuais podem ser deixadas facilmente porque alguém tem de optar por ir lá e, por isso, podem escolher que ora não. Comunidades virtuais podem ser de vários tipos diferentes, a partir de notícias de discussões sobre um tema limitado permitindo, nas relações virtuais, todas as versões off-line sociais (JORDAN, 1999:100). (6)

Ao longo de todo o ecossistema digital, este movimento está crescendo e tais características aplicam-se ao ciberespaço. Para Benkler, neste espaço pós-moderno:

coisas podem acontecer, vinculadas a qualquer tema, onde e, em qualquer momento, as capacidades das pessoas se potencializam pelos meios de reprodução, difusão, e, ao se comunicarem em seus fóruns, podem, inclusive, mudarem a relação que tinham com os acontecimentos que os rodeiam (BENKLER, 2006: 8).

$\mathrm{Na}$ verdade, esta divisão entre comunidades on-line e as tradicionais, Warschauer destaca que, "de fato, ela não existe". Existem muitas pontes comunicacionais entre elas, através da partilha de dados virtuais e reais. Como exemplos podem ser cartas, fax, telefone, livros e outros meios de comunicação social. Todos os 
membros de comunidades diferentes têm informações compartilhadas em um intervalo de espaço geográfico em indistintas formas e este intercâmbio de dados não acontece só na ágora virtual; neste caso, a palavra ainda funciona como uma ferramenta importante para os indivíduos ditos virtuais.

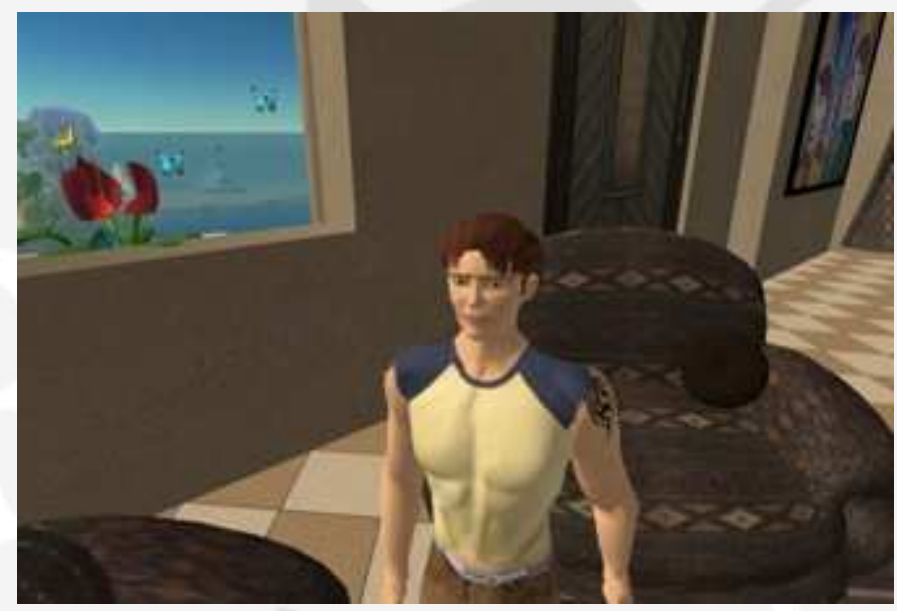

Figura 7 - Um exemplo de avatar

Contudo, apesar da importância da palavra, o projeto Second Life (SL) - famoso que deu origem ao modismo tecnológico na web - por mais complicado que possa parecer, seu sucesso se justifica, principalmente, pela dimensão das três concepções do espaço, ou ainda, a ilusão 3D; é a sedução da interface gráfica e o ambiente de games que tem imperado nas relações on-line do projeto. Afinal, muitos esforços foram demonstrando pelo designer do SL, Philip Rosedale, a fim de fascinar a sua nação virtualizada. Disponibilizado por meio de textos, imagens, o SL oferece também ambientes interativos; ao contrário das CVs comuns, espalhadas ao longo de todo o ecossistema digital, o projeto legitimou a conhecida relação dos usuários para com o uso da linguagem dos jogos não só por palavras como o IRC, os MOOs, os MUDS (Turke, 1997), mas por imagens também. Dentro do SL, aos usuários abriu-se a possibilidade de viver o seu avatar. Jones enfatiza que o significante avatar deriva do sânscrito "avatara" e destina-se a sugerir "a idéia de uma espécie de transubstanciação, a encarnação da vida sob uma forma diferente" (Tofts, 2003: 56).

De 2003 pra cá, outro site de relacionamento, o celebrado Orkut - que pertence à gigante Google - surge como um empreendimento de sucesso e tem informado sempre um número expressivo de usuários. Foi um boom das comunidades digitais, e colocou o Brasil como o primeiro país onde o seu sucesso se tornou mais evidente, seguidos pelos Estados Unidos e Índia. Embora bem sucedido, o Orkut tem se envolvido em vários problemas com a justiça desses países.

$\mathrm{Na}$ seqüência, outro site de relacionamento surgiu, cujo nome é MySpace. O seu sucesso foi também instantâneo e tendo sido eleito como uma das melhores redes sociais. De acordo com a Alexa Internet (www.alexa.com), o MySpace é atualmente o quinto mais popular do mundo de língua inglês e em qualquer idioma, além do terceiro mais conhecido nos Estados Unidos.

Afinal, se a rede tem caráter social, seus programadores, web designers, editores on-line pensam o impacto destes serviços na cibersociedade, ou é apenas mercado de mídia? Onde pode estar localizada a fronteira entre a comunicação social e o mercado neste ecossistema digital? Não é fácil responder a estas perguntas, e mais virão... Entretanto, visualizamos tão-somente como esses sites tem manipulado e maquiado "a idéia de socialização"? A propósito, é possível contar o número de amigos que temos e mostrar-lhe em frente a uma página? Será esta a verdadeira expressão de amizade em redes sociais? Que tipo relacionamento que estamos 
construindo na web? Como já apontado, não apenas a construção do "ser" encontra-se fragmentada na tela (Turkle), mas a sua concepção? E ela é, portanto, uma montagem?

Ainda há sete anos, uma dos mais conhecidas pesquisas na campo da comunidade virtual, Netville em Toronto, Canadá, conduzida por Hampton \& Wellman deixou-nos estas questões:

Podemos apoiar, sociável e significativas relações on-line? Vida on-line irá substituir, complementar, ou a completar a vida real? Netville está localizado em um subúrbio residencial planejado em Toronto equipados com uma rede de alta velocidade, como parte da sua concepção (...) (WELLMAN \& HAMPTON apud ISBISTER \& ISHIDA, 2000: 194-208). (7)

As respostas a tais perguntas, naquele tempo, como enfatiza Castells, foram positivas e, hoje, contudo, precisariam ser talvez retomadas pois novos desafios se apresentam.

\section{Mercados e máquinas}

É indubitável que os mercados e as máquinas muito representam na compreensão do fluxo de acesso à rede de rede de computadores, e não seria necessário citar as origens do sistema de máquinas envolvido nele. Todavia, vender e/ou comprar nas arenas digitais não parecem acalentar grandes diferenças. No entanto, a maneira como nós estamos realizando estas atividades também está mudando. Hoje, forma colaborativas dominam tais relações, como destacam Tapscott \& Williams (2007):

Devido às profundas mudanças na tecnologia, demografia, negócios, a economia, e no resto do mundo, estamos entrando numa nova era em que as pessoas participam na economia como nunca antes. Esta nova participação chegou a um ponto onde buscam novas formas de colaboração em massa estão mudando o modo como produtos e serviços são inventados, produzidos, comercializados e distribuídos numa esfera global. Esta mudança de longo alcance apresenta oportunidades para todas as empresas e para cada pessoa que se conecta (TAPSCOTT \& WILLIAMS, 2007: 10) (8).

Esses autores alcunharam a expressão wikinomics para estas novas formas de relação comercial e os seus antecedentes possuem quatro princípios: o de ser aberto, partilhado, agir globalmente, e num sistema ponto-aponto, ou seja, com a rede de máquinas consolidando o processo. E nesse ritmo, o jornalismo colaborativo na rede, - wiki - , vem encontrando novos atalhos junto às RSS, feeds etc.

\section{Considerações finais}

Nesta relação de redes em convergência, muitos dos fenômenos da mídia on-line podem ser bem mais compreendidos, contudo, ainda carecemos de ferramentais das ciências sociais aplicadas, e, aí, desponta a comunicação - através de pesquisas cibersociais - para alavancar novos direcionamentos teórico-práticos nas arenas da mídia on-line.

É claro que um artigo não poderia esgotar as características do gap que separa as práticas das teorias apresentadas, mas se coloca como fomento às novas discussões, em vista da solidificação na busca de instrumentos que, só concebidos à luz da convergência entre as redes - por hora introduzidas -, podem delinear o novo perfil deste ecossistema digital. Quiçá a tríade, destacada por Kerchkhove, tem vindo a enfatizar ainda mais o traço fronteiriço entre a cidadania clássica e a on-line, onde suas constantes interações arquitetam o novo cibercidadão e sua produção jornalística on-line. 


\section{Bibliografia:}

AUGÉ, Marc. Não-lugares - introdução a uma antropologia da supermodernidade. Campinas: Papirus, 1994.

ANDERSON, Benedict. Imagined communities: reflections on the origin and spread of nationalism, 1983.

BARNEY, Darin. One nation under google - citizenship in the technological Republic. Toronto: UofT/Hart House Lecture Press, 2007.

BAUMANN, Zygmunt. Identidade. Rio de Janeiro: Jorge Zahar, 2005.

BENKLER, Yonchai. The wealth of networks - how social production transforms market and freedom. New York: Haven and London, 2006.

CRAPTON, Jeremy W. The political mapping of cyberspace, 2003.

RHEINGOLD, Howard. The virtual community: Homesteading on the electronic frontier. 2nd ed. Cambridge, MA: MIT Press, 2000.

. Smart Mobs: The Next Social Revolution. Cambridge, MA: Perseus, 2002.

ISBISTER, Katherine \& ISHIDA, Toru (eds.). Digital cities: technologies, experiences, and future perspective. Lecture Notes in Computer Science 1765, Heidelberg, Germany, Springer-Verlag, 2000.

JONES, Donald E. Avatar: Constructions of Self and Place in Second Life and the Technological Imagination. Georgetown University: Communication, Culture and Technology, 2007.

JORDAN, Tim. Cyberpower: the culture and politics of cyberspace and the internet. London and New York, 1999.

KERKCHOVE, Derrick de. The architecture of intelligence - the information technology revolution in Architecture. Birkhauser, Publishers for Architecture, Switzerland, 2001.

MCLUHAN, Marshall. The Gutenberg Galaxy: the Making of Typographic Man. Toronto: University of Toronto Press, 1962.

NICOLA, Ricardo. Cibersociedade - quem é você no mundo on-line? São Paulo: Senac, 2004.

NICOLA, Ricardo.”Cibercidadania na República Tecnológica: contribuições info-inclusivas dos novos paradigmas transculturais canadenses". XXX Congresso Brasileiro de Ciências da Comunicação - Sociedade Brasileira de Estudos Interdisciplinares da Comunicação, 2007.

PAGON, Elia Patricia Pekica. GLOBISH the communication of the future. France, Jean-Paul Nerrière/GNU Public License, 2004. Access at: http://www.jpn-globish.com/articles.php?lng (April 20th, 2007).

TAPSCOTT, Don \& WILLIAMS, Anthony D. Wikinomics - how mass collaboration changes everything. New York: McGraw-Hill, 2007.

TURKLE, Sherry. Life on the screen - the identity in the age of the Internet, 1997. 
TOFTS, D. “Avatars of the Tortoise: Life, Longevity and Simulation”. Digital Creativity, 14.1, 54-63, 2003.

WARSCHAUER, Mark. Technology and social inclusion - rethinking the digital divide, 2004.

\section{Webgrafia}

www.alexa.com

http://midia.press.sites.uol.com.br

www.well.com

www.utoronto.ca/mcluhan/

www.mcluhan.utoronto.ca/academy/mms1

http://www.donthideit.com/profiles/darryl.html

http://www.cdt.org/

http://www.albion.com/netiquette/index.html

http://www.realtime-websecurity.com/articles_and_analysis/2006/10/good_cybercitizen ship_or_selfi.html http://fr.takingitglobal.org/express/panorama/article.html?ContentID=6533\&start=0

\section{Notas:}

Trabalho apresentado ao $6^{\circ}$ Encontro Nacional de Pesquisadores em Jornalismo $\left(6^{\circ}\right.$ SBPJor) e ao XXXI Congresso Brasileiro de Ciências da Comunicação (Intercom). Escrito originalmente em inglês.

(1) Do original: "The architeture of intelligence is the archictecure of connectivity. It is the architecture that bring together the three main spatial environments that we live in and with today: mind, world, and networks"(Kerckhove, The Architeture of Inteligence - the information revolution in Architecture, p. 7).

(2) Idem, 2004.

(3) Do original em inglês: “(...) internet access is starting to reach saturation point in the United States but is just taking off in many other countries around the world. As a critical mass of users gets online in a particular language, more people and businesses create web sites in that language, and speakers of language also have a greater number of potential partners for computer-mediated communication. This trend is also accelerated by the expansion of operating systems and Web page authoring software in non-Roman scripts, which allows people to communicate more easily in non-alphabetic languages such as Japanese, Chinese, and Hebrew. Because of these trends, the proportion of Web sites in English is expected to drop to $40 \%$ in the next decade (Graddol, 1997)" (WARSCHAUER, 2004: 12).

(4) Do original em inglês: “(...) The goal of a Digital Nation is not just to do things faster; it's to do them differently, revolutionizing the way we educate people, deliver health care, and engage in productive work. Implementing a Digital Nation vision will save the country billions of dollars over the coming years and will 
greatly increase quality of life as technology expands choice and opportunity. In the arenas of health delivery and e-work alone, information and communications technologies invite us to refresh our approach to rural medicine and traffic alleviation (...)" (WILHELM, 2004: xii).

(5) Do original em inglês: "Cyberspace for us now is a kind of mapping into which we project our fears and hopes: it is our 'project'. As such a projection, cyberspace presents a number of representational (and thus cartographic) issues. It is no accident that all maps are projections. The issues are not in mapping its abstractness (cartography has mapped the abstract for centuries) but with its spatial politics. Cyberspace is a classic case of a space which is produced, and which in turn produces (spatialized) subjectivity. Thus not only can cyberspace be mapped, but in tracing out its contours we are tracing out the lines on our own faces. Cyberspace is an area of geographic knowledge that sits equally between society and technology. It invites the 'fieldwork' for a critical politics of spatial representation and from that a critical politics of geography and space" (CRAPTON, 2003: 6).

(6) Do original em inglês: "Communities emerge in cyberspace when a number of users create avatars that return again and again to the same informational space. Individuals may find they are no longer alone in cyberspace but have developed relations with a number of other stable avatars and have become part of a virtual community. Virtual communities can be left easily because someone must choose to go there and so can choose not to. Virtual communities can be of many different types, from newsgroup discussions about a limited topic to MUDs that allow virtual versions of all offline social relations" (JORDAN, 1999: 100).

(7) Do original em inglês: "Can supportive, sociable and meaningful relations be maintained on-line? Will life on-line replace, complement, or supplement life in the flesh? Netville is a residential development located in suburban Toronto equipped with a high-speed network as part of its design (...). In: WELLMAN, Barry \& HAMPTON, Keith N. Examining community in the digital neighborhood early results from Canada's wired suburb (ISBISTER \& ISHIDA, 2000: 194-208).

(8) Do original em inglês: "Due to deep change in technology, demographics, business, the economy, and the world, we are entering a new age where people participate in the economy like never before. This new participation has reached a tipping point where new forms of mass collaboration are changing how goods and services are invented, produced, marketered, and distributed on a global basis. This change presents farreaching opportunities for every company and for every person who gets connected" (TAPSCOTT \& WILLIAMS, 2007: 10).

\section{Mini Currículo :}

Doutor em Multimeios pela Unicamp, é professor doutor da Faculdade de Arquitetura, Arte e Comunicação (Unesp-Bauru). Autor de Cibersociedade - quem é você no mundo on-line (Senac-SP, 2004), tendo realizado pós-doutorado (2006-2007) no McLuhan Program in Culture and Technology da Universidade de Toronto, como bolsista Capes. E-mail: midia.press@uol.com.br 